\title{
Pediatric Acute Kidney Injury to the Subsequent CKD Transition
}

\author{
Fang Wang Jie Ding \\ Department of Pediatrics, Peking University First Hospital, Beijing, China
}

\section{Keywords}

Acute kidney injury - Chronic kidney disease · Children .

Epidemiology · Biomarkers

\begin{abstract}
Background: Both acute kidney injury (AKI) and CKD are complex syndromes caused by multiple etiologies and presented with various degrees of severity. Studies on adults provide strong evidence that $\mathrm{AKI}$ is an independent risk factor for both the initiation and progression of CKD, and the severity, frequency, and duration of AKI are crucial factors in the subsequent development of CKD. However, without consensus definitions of AKI and CKD and long-term followup studies using predictive biomarkers, it is difficult to clarify the potential for transition from AKI to CKD in pediatric populations. The goal of this review is to describe the most recent studies in epidemiology of pediatric AKI and biomarkers aiding in the earlier detection of AKI and CKD. Summary: KDIGO criteria for AKI have been widely applied for pediatric AKI studies. AKI in critically ill and non-critically ill children is common. CKD is highly prevalent in pediatric AKI survivors. Compared with traditional biomarkers such as serum $\mathrm{Cr}$, proteinuria, and estimated glomerular filtration rate, urinary biomarkers earlier identifying AKI may also detect CKD earlier, but additional studies are required to determine their clinical utility. Key Messages: The use of consensus AKI cri-
\end{abstract}

karger@karger.com www.karger.com/kdd

Karger $\stackrel{\text { ' }}{5}$

GOPEN ACCESS
(C) 2020 The Author(s)

Published by S. Karger AG, Basel

This article is licensed under the Creative Commons AttributionNonCommercial-NoDerivatives 4.0 International License (CC BYNC-ND) (http://www.karger.com/Services/OpenAccessLicense) Usage and distribution for commercial purposes as well as any distribution of modified material requires written permission. teria has improved our understanding of pediatric AKI epidemiology, and an association between AKI and CKD in pediatric populations has been endorsed. However, further studies are needed to better answer a definitive causal relationship between pediatric AKI and the subsequent development of CKD.

(C) 2020 The Author(s)

Published by S. Karger AG, Basel

\section{Introduction}

Either acute kidney injury (AKI) or CKD is a clinical syndrome featured by alterations in kidney function or structure. With the prevalence of both situations increasing, they are becoming worldwide serious public health problems. Mounting evidence in adults suggests that AKI is an independent risk factor for both the initiation and progression of CKD [1-5], and the severity, frequency, and duration of AKI are crucial factors in the AKI to CKD transition. Although the mechanisms underlying progression from AKI to CKD in humans are not completely understood, studies in animals demonstrate that a maladaptive cellular repair mediates the AKI to CKD transition, which involves hypoxia and microvascular rarefaction, alterations in renal-resident cell phenotypes and functions, cell cycle arrest in the G2/M phase, persistent chronic inflammation, and development of intersti- 
tial fibrosis, mitochondrial dysfunction and fragmentation, epigenetic changes, renin-angiotensin system (RAS) activation, and cell and tissue aging [6-13]. However, the potential for transition from AKI to CKD in pediatric populations is still under investigation. This review focuses on the most recent studies in epidemiology of pediatric $\mathrm{AKI}$ and biomarkers aiding in the earlier detection of $\mathrm{AKI}$ and $\mathrm{CKD}$.

\section{AKI Epidemiology and AKI to CKD in Pediatric Populations}

AKI is a complex syndrome caused by multiple etiologies and presented with various degrees of severity. Variation in the most common etiologies of AKI in hospitalized children had been reported. For instance, the Assessment of Worldwide Acute Kidney Injury, Renal Angina, and Epidemiology (AWARE) study, including a cohort of 4,683 children from 32 intensive care units, showed strongest independent association between severe AKI (defined as stage 2 or 3 AKI according to the complete KDIGO criteria) and cardiovascular and respiratory diseases [14], whereas Madsen et al. [15] found that about one-third of 382 patients (younger than 15 years) undergoing cardiac surgery for congenital heart disease experienced AKI within 5 days of surgery, and Koh and colleagues [16] demonstrated two-thirds of 1,057 pediatric and adolescent patients developed AKI within 100 days post-hematopoietic cell transplantation.

The long-standing lack of a consensus AKI definition hampered pediatric AKI studies. Fortunately, there are 3 sets of pediatric AKI diagnostic criteria: pRIFLE [17], AKIN [18], and KDIGO [19], and KDIGO harmonized the existing definitions. A retrospective, electronic medical record-enabled, single-center study including a cohort of 14,795 hospitalized children showed AKI incidence and staging were different based on pRIFLE, AKIN, and KDIGO: pRIFLE identified the largest AKI and stage 1 cohorts, AKIN and KDIGO detected similarly sized AKI and stage 1 and 3 cohorts, and AKIN resulted in the smallest stage 3 cohort [20]. Nevertheless, currently, KDIGO criteria have been widely applied for pediatric AKI studies.

The incidence of AKI in pediatric populations is one of the critical factors to understand the burden of AKI. In the AWARE study, AKI developed in $26.9 \%$ and severe AKI (KDIGO stage $2 / 3$ ) in $11.6 \%$ of patients during the first week of hospitalization [14]. Perhaps, this study demonstrated the most accurate incidence of AKI in critically ill children from developed countries. Other spe- cific conditions such as pediatric diabetic ketoacidosis with AKI have been concerned recently. In a retrospective study, among 165 diabetic ketoacidosis admissions in a tertiary pediatric hospital, the researchers found that AKI (defined by the KDIGO creatinine criteria) occurred in $64.2 \%$ of all cases, and $65.1 \%$ patients had KDIGO stage $2 / 3$ [21]. In addition, AKI is common in non-critically ill children. A retrospective single-center study involving 13,914 general pediatric admissions showed that at least $5 \%$ of admissions developed AKI [22]. Recently, a multicenter retrospective study involving 101,836 pediatric inpatients from China, using the KDIGO creatinine criteria, demonstrated the incidence of community-acquired AKI was 7\% and that of hospital-acquired AKI was 13\% [23]. Perhaps, this study represented the largest epidemiologic study of pediatric AKI in developing countries. It is worth noting that this study showed only $4 \%$ of AKI events were diagnosed on the discharge records, suggesting the underestimation of $\mathrm{AKI}$, and it is urgent to raise the awareness of pediatric AKI among physicians for improving health care in children.

A number of clinical investigations have demonstrated that CKD is highly prevalent in pediatric AKI survivors following cardiac surgery (mostly), stem cell transplant, septic shock, high nephrotoxic medication exposure, and so on [24]. However, chronic renal injury was defined as the presence of proteinuria, hypertension, estimated glomerular filtration rate $<90 \mathrm{~mL} / \mathrm{min} / 1.73 \mathrm{~m}^{2}$ or $<60 \mathrm{~mL} /$ $\min / 1.73 \mathrm{~m}^{2}$, and hyperfiltration, that is, to say lack of a consensus definition of CKD, limited our ability to compare analyses and extrapolate findings of the available data. On the contrary, an association between AKI and CKD was not found in 2 longitudinal studies assessing CKD in pediatric cardiac surgery-related AKI survivors $[25,26]$, whereas persistently elevated urinary biomarkers of kidney injury were observed. One study evaluated 5 -year renal outcomes among 131 children who underwent cardiac surgery for congenital cardiac defects [25]. Fifty-seven children developed postoperative AKI (defined according to serum $\mathrm{Cr}$ level or longer hospital stays), and 5-year CKD (defined as estimated glomerular filtration rate $<90 \mathrm{~mL} / \mathrm{min} / 1.73 \mathrm{~m}^{2}$ or microalbuminuria) was present in 3 children with AKI and 18 children without AKI. Another study demonstrated evidence of chronic kidney injury (including estimated glomerular filtration rate, proteinuria, and blood pressure measurement) at 7-year follow-up after cardiac surgery did not differ between 33 children who developed AKI (defined according to serum $\mathrm{Cr}$ ) and 18 children who did not develop AKI at the time of reparative surgery. 


\section{Noninvasive Biomarkers for Monitoring AKI to CKD Progression}

One of the greatest challenges for the treatment of AKI and CKD is identification of them in a timely manner. Serum Cr concentration, despite the well-known imperfect biomarker, remains the most common surrogate biomarker of kidney function. Fortunately, owing to advances in understanding the pathophysiology of both AKI and $\mathrm{CKD}$, a number of promising markers for detecting earlier kidney injury and prognosticating kidney disease progression have been developed. For example, a test based on the combination of urinary concentrations of tissue inhibitor of metalloproteinase 2 and insulin-like growth factor-binding protein 7 has been approved for the specific identification of AKI in patients beyond 21 years, although its low predictive value is an important issue. The performance of such biomarkers in children is briefly mentioned below.

A considerable amount of clinical investigations demonstrated that urinary biomarkers of tubular damage such as neutrophil gelatinase-associated lipocalin (NGAL), kidney injury molecule-1 (KIM-1), interleukin-18 (IL-18), and liver-type fatty acid-binding protein (L-FABP) are potential markers for early detection of AKI before serum $\mathrm{Cr}$ rises in different settings. Interestingly, inconsistent results were drawn from 2 longitudinal studies assessing the association between urinary biomarkers NGAL, KIM-1, IL-18, and L-FABP and chronic renal injury in pediatric AKI survivors following cardiac surgery using cardiopulmonary bypass. Cooper et al. [26] found that 7-year elevated urinary concentrations of IL18, KIM-1, and L-FABP were detected in children with postoperative AKI, although these children did not present with conventional signs of CKD. Greenberg et al. [27] found that there was no association between postoperative AKI, CKD, and hypertension and 5-year urinary concentrations of IL-18, KIM-1, and NGAL. Therefore, the clinical relevance of urinary concentrations of NGAL, KIM-1, IL-18, and L-FABP in assessments for potential long-term kidney consequences of other condition-associated pediatric $\mathrm{AKI}$ is required.

Recent in vitro and in vivo studies demonstrated RAS activation involved in the pathogenesis of AKI-CKD transition [12]. The key substrate for the RAS is angiotensinogen (AGT), and renal tubular cells can produce AGT. Emerging evidence suggests that urinary AGT is an encouraging biomarker to predict $\mathrm{AKI}$ or $\mathrm{CKD}$ progression [28]. Using a murine bilateral renal ischemia/reperfusion injury model and a prospective cohort study of 20 pa- tients with acute tubular necrosis, Cui and colleagues found that urinary AGT can dynamically detect renal structural recovery following AKI [29]. However, additional studies are required to determine urinary AGT ability to predict early pediatric AKI-CKD transition.

\section{Conclusions}

Hospitalized children develop AKI frequently, and this disease is associated with adverse outcomes. Recently, the use of consensus AKI criteria has improved our understanding of pediatric AKI epidemiology, and an association between AKI and CKD in pediatric populations has been endorsed. However, long-term, large-scale prospective studies using a consensus definition of CKD and encouraging kidney injury biomarkers are needed to better answer a definitive causal relationship between pediatric AKI and the subsequent development of CKD. In adults, specific therapeutic approaches to prevent the transition from AKI to CKD are still not available.

\section{Conflict of Interest Statement}

The authors have no conflicts of interest to declare.

\section{Funding Sources}

Supported by Beijing Key Laboratory of Molecular Diagnosis and Study on Pediatric Genetic Diseases (No. Z141107004414036).

\section{Author Contributions}

F.W. drafted the manuscript. J.D. contributed to supervision and writing - review and editing.

\footnotetext{
References

1 Coca SG, Singanamala S, Parikh CR. Chronic kidney disease after acute kidney injury: a systematic review and meta-analysis. Kidney Int. 2012;81(5):442-8.

2 Heung M, Steffick DE, Zivin K, Gillespie BW, Banerjee T, Hsu CY, et al. Acute kidney injury recovery pattern and subsequent risk of CKD: an analysis of veterans health administration data. Am J Kidney Dis. 2016;67(5):742-52.

3 Kim CS, Bae EH, Ma SK, Kweon SS, Kim SW. Impact of transient and persistent acute kidney injury on chronic kidney disease progression and mortality after gastric surgery for gastric cancer. PLoS One. 2016;11(2): e0168119.
}

Wang/Ding 
4 Palomba H, Castro I, Yu L, Burdmann EA. The duration of acute kidney injury after cardiac surgery increases the risk of long-term chronic kidney disease. J Nephrol. 2017;30(4): 567-72.

5 Hatakeyama Y, Horino T, Nagata K, Matsumoto T, Terada Y, Okuhara Y. Transition from acute kidney injury to chronic kidney disease: a single-centre cohort study. Clin Exp Nephrol. 2018;22(6):1281-93.

6 Chawla LS, Kimmel PL. Acute kidney injury and chronic kidney disease: an integrated clinical syndrome. Kidney Int. 2012;82(5): 516-24.

7 Chawla LS, Eggers PW, Star RA, Kimmel PL. Acute kidney injury and chronic kidney disease as interconnected syndromes. N Engl J Med. 2014;371(1):58-66.

8 Venkatachalam MA, Weinberg JM, Kriz W, Bidani AK. Failed tubule recovery, AKI-CKD transition, and kidney disease progression. J Am Soc Nephrol. 2015;26(8):1765-76.

9 Fiorentino M, Grandaliano G, Gesualdo L, Castellano G. Acute kidney injury to chronic kidney disease transition. Contrib Nephrol. 2018;193:45-54.

10 Strausser SA, Nakano D, Souma T. Acute kidney injury to chronic kidney disease transition: insufficient cellular stress response. Curr Opin Nephrol Hypertens. 2018;27(4):314-22.

11 García-Ortuño LE, Araceli Bobadilla N. Integrative view of the mechanisms that induce acute kidney injury and its transition to chronic kidney disease. Rev Invest Clin. 2018; 70(6):261-8.

12 Chou YH, Chu TS, Lin SL. Role of renin-angiotensin system in acute kidney injurychronic kidney disease transition. Nephrology. 2018;23(Suppl 4):121-5.

13 Nangaku M, Hirakawa Y, Mimura I, Inagi R, Tanaka T. Epigenetic changes in the acute kidney injury-to-chronic kidney disease transition. Nephron. 2017;137(4):256-9.
14 Kaddourah A, Basu RK, Bagshaw SM, Goldstein SL; AWARE Investigators. Epidemiology of acute kidney injury in critically ill children and young adults. Ann Clin Biochem. 2017;54(3):416-20.

15 Madsen NL, Goldstein SL, Frøslev T, Christiansen CF, Olsen M. Cardiac surgery in patients with congenital heart disease is associated with acute kidney injury and the risk of chronic kidney disease. Kidney Int. 2017; 92(3):751-6.

16 Koh KN, Sunkara A, Kang G, Sooter A, Mulrooney DA, Triplett B, et al. Acute kidney injury in pediatric patients receiving allogeneic hematopoietic cell transplantation: incidence, risk factors, and outcomes. Biol Blood Marrow Transplant. 2018;24(4):758-64.

17 Akcan-Arikan A, Zappitelli M, Loftis LL, Washburn KK, Jefferson LS, Goldstein SL. Modified RIFLE criteria in critically ill children with acute kidney injury. Kidney Int. 2007;71(10):1028-35.

18 Mehta RL, Kellum JA, Shah SV, Molitoris BA, Ronco C, Warnock DG, et al. Acute kidney injury network: report of an initiative to improve outcomes in acute kidney injury. Crit Care. 2007;11(2):R31.

19 Kellum JA, Lameire N, Aspelin P, Barsoum RS, Burdmann EA, Goldstein SL, et al. Kidney disease: improving global outcomes (KDIGO) acute kidney injury work group. KDIGO clinical practice guideline for acute kidney injury. Kidney Int. 2012;2(Suppl 2):1-138.

20 Sutherland SM, Byrnes JJ, Kothari M, Longhurst CA, Dutta S, Garcia P, et al. AKI in hospitalized children: comparing the pRIFLE, AKIN, and KDIGO definitions. Clin J Am Soc Nephrol. 2015;10(4):554-61.

21 Hursh BE, Ronsley R, Islam N, Mammen C, Panagiotopoulos C. Acute kidney injury in children with type 1 diabetes hospitalized for diabetic ketoacidosis. JAMA Pediatr. 2017; 171(5):e170020.
22 McGregor TL, Jones DP, Wang L, Danciu I, Bridges BC, Fleming GM, et al. Acute kidney injury incidence in noncritically ill hospitalized children, adolescents, and young adults: a retrospective observational study. Am J Kidney Dis. 2016;67(3):384-90.

23 Xu X, Nie S, Zhang A, Mao J, Liu HP, Xia H, et al. Acute kidney injury among hospitalized children in China. Clin J Am Soc Nephrol. 2018;13(12):1791-800.

24 Sigurjonsdottir VK, Chaturvedi S, Mammen C, Sutherland SM. Pediatric acute kidney injury and the subsequent risk for chronic kidney disease: is there cause for alarm? Pediatr Nephrol. 2018;33(11):2047-55.

25 Greenberg JH, Zappitelli M, Devarajan P, Thiessen-Philbrook HR, Krawczeski C, Li S, et al. Kidney outcomes 5 years after pediatric cardiac surgery: the TRIBE-AKI study. JAMA Pediatr. 2016;170(11):1071-8.

26 Cooper DS, Claes D, Goldstein SL, Bennett MR, Ma Q, Devarajan P, et al. Follow-up renal assessment of injury long-term after acute kidney injury (FRAIL-AKI). Clin J Am Soc Nephrol. 2016;11(1):21-9.

27 Greenberg JH, Devarajan P, Thiessen-Philbrook HR, Krawczeski C, Parikh CR, Zappitelli M; TRIBE-AKI Consortium. Kidney injury biomarkers 5 years after AKI due to pediatric cardiac surgery. Pediatr Nephrol. 2018;33(6):1069-77.

28 Connor KL, Denby L. Urinary angiotensinogen as a biomarker for acute to chronic kidney injury transition: prognostic and mechanistic implications. Clin Sci. 2018;132(21):2383-5.

29 Cui S, Wu L, Feng X, Su H, Zhou Z, Luo W, et al. Urinary angiotensinogen predicts progressive chronic kidney disease after an episode of experimental acute kidney injury. Clin Sci. 2018;132(19):2121-33. 